\title{
6 Minute walk test and idiopathic pulmonary fibrosis: distance or desaturation? A prospective observational study
}

\author{
Neeraj Gupta ${ }^{1}$, Mukesh Goyal ${ }^{1 *}$, Harish $V_{K}{ }^{2}$, Arjun Chandran ${ }^{1}$, and Amit Sharma ${ }^{1}$ \\ ${ }^{1}$ Department of Respiratory Medicine, JLN Medical College, Ajmer, Rajasthan, India \\ ${ }^{2}$ Department of Respiratory Medicine, Indira Gandhi Medical College and Research Institute, Puducherry, India
}

\begin{abstract}
Background: 6MWT is simple, easy to perform, highly reproducible test. Its precise indication is to assess the response to therapy in various chronic lung disorders. High cost and inaccessibility of diffusion measurements in most of the clinical settings brings the utility of this test in top priority in routine follow-up of patients suffering from ILDs. However, results in various studies are not the same.

Material and Methods: 17 patients with Idiopathic Pulmonary Fibrosis were included in the study. They were assessed clinically, radiologically and by complete pulmonary function tests at initial presentation. They were all treated with same protocol with standard doses of pirfenidone, low dose corticosteroid and $\mathrm{N}$-Acetyl Cysteine. A six-month follow-up study with same protocol was performed. The parameters of clinical importance were NYHA Dyspnea grading, $6 \mathrm{MWT}$, FVC and $\mathrm{DL}_{\mathrm{CO}}$. The statistical analysis was performed to assess the clinical significance.

Results: In contrast to some of the quoted studies, we found that $6 \mathrm{MWD}$ is not the sole parameter to be relied upon in patients with IPF treated with pirfenidone containing regimes as it did not change significantly over 6 months' follow-up. Rather it was the baseline oxygen saturation and desaturation after $6 \mathrm{MWT}$ that carries a prognostic significance in this group of patients. Furthermore, its trend during 6 months closely followed the trends in change in grade of dyspnea, $\mathrm{FVC}$ and $\mathrm{DL} \mathrm{CO}_{\mathrm{C}}$

Conclusion: Inclusion of oxygen saturation studies in 6MWT carries a prognostic significance and must be an integral part of physiological parameters to be followed in patients with IPF.
\end{abstract}

\section{Abbreviations}

6MWT: Six Minute Walk Test; 6MWD: Six Minute Walk Distance; IPF: Idiopathic Pulmonary Fibrosis; ILD: Interstitial Lung Diseases; FVC: Forced Vital Capacity; $\mathrm{DL}_{\mathrm{CO}}$ : Diffusion Capacity of Lungs; NYHA: New York Heart Association

\section{Introduction}

6 MWT is a simple test, easy to perform, better tolerated and more reflective of day to day activities than other walk tests [1]. Since more of the activities of daily living are performed at sub-maximal levels of exertion, the 6MWD may better reflect the functional exercise levels for daily physical activities and formal measures of quality of life [2]. The strongest indication for 6MWT is for measuring the response to medical intervention in patients with moderate to severe heart or lung disease. Changes in $6 \mathrm{MWD}$ after therapeutic interventions correlate with subjective improvement in dyspnea $[3,4]$. The test has a better reproducibility and lesser short term variability.

A diagnosis of IPF is associated with a median mortality of approximately 3 years. According to ATS/ERS Guidelines (2013), every 6-12 months follow up is necessary in case of ILD patients. It includes a full work up of PFTs including spirometry, diffusion capacity and 6MWT apart from HRCT chest and clinical evaluation. Small changes in $\mathrm{FVC}$ and $\mathrm{DL}_{\mathrm{CO}}$ within first 6 to 12 months of observation may translate into major survival differences during long term follow-up [5].

We performed 6MWT in all of our patients of IPF-

1. As a protocol at the time of diagnosis and 6 monthly follow-up along with other clinical, radiological and functional parameters.
2. Because the original ATS Statement [6] does not include ILD as an indication for 6MWT, only isolated studies are available in the literature.

3. Pulse oximetry is considered as an optional parameter in $6 \mathrm{MWT}$, distance being the primary outcome measure. We did pulse oximetry based on the concept that improvement in $\mathrm{SpO}_{2}$ (peripheral capillary oxygen saturation) during serial evaluation may be manifested either by an increased distance or reduced symptoms with same distance walked [3].

While going through various studies done in the past using 6MWT in patients with IPF, we found that some of them did not observe any significant change in $6 \mathrm{MWD}$ over the time in these patients while few others opined that a fall in 6MWD was associated with increased mortality (discussed later). Such a difference in opinion in these studies and our observations in patients with IPF prompted us to share our results and views.

\section{Material and methods}

The study was carried out in the Department of Respiratory Medicine, Jawahar Lal Nehru Medical College, Ajmer. 17 consecutive cases of IPF consistent with diagnosis of UIP on HRCT (high resolution

Correspondence to: Dr. Mukesh Goyal, Assistant Professor, Department of Respiratory Medicine JLN Medical College, Ajmer, Rajasthan, India

Received: February 20, 2017; Accepted: March 23, 2017; Published: March 27, 2017 
computerised tomography) Chest were included in the study after informed consent. After detailed clinical history including NYHA grading of dyspnea [7] and physical examination, all these patients were subjected to complete pulmonary function tests including spirometry, $\mathrm{DL}_{\mathrm{CO}}$ and $6 \mathrm{MWT}$ as per standard protocol. Oxygen saturation was recorded in all the patients during 6MWT along with 6MWD. Desaturation during 6MWT was also observed.

All these patients were treated with standard doses of Pirfenidone (200mg tablets, 1 tablet thrice a day for one week followed by 2 tablets thrice a day for $2^{\text {nd }}$ week and 3 tablets thrice a day from $3^{\text {rd }}$ week onwards), low dose corticosteroids (10mg prednisolone daily) and $\mathrm{N}$-Acetyl Cysteine (600 mg thrice a day). All these patients were under regular follow-up and were re-evaluated at the end of 6 months with the same protocol. No significant dose limiting side effects were observed. The results were recorded, tabulated and statistically analysed.

\section{Results}

Our study included 17 cases of IPF, out of which 14 completed the study while 3 patients died. All the three patients who died during the study had a baseline $6 \mathrm{MWD}$ of less than 126 meters and $\mathrm{DL}_{\mathrm{CO}}$ of less than $35 \%$ at the time of initial presentation. The overall results are depicted in tables 1 to 4 . The data are clearly evident and reveal that although there was an increase in mean 6MWD in the study population (144 meters at initial presentation and 151.79 meters at the end of 6 months), it was statistically insignificant, $p$ value was 0.967 . In other words, it was interpreted that although mean distance walked in 6 minutes by the subjects did not changed significantly but the FVC, $\mathrm{DL}_{\mathrm{CO}}$, grading of dyspnea and Desaturation during 6MWT consistently fell which were statistically significant.

Table 1 shows demographic characteristics of 17 patients. Mean age of the study population was 63 years with male to female ratio of 2.4:1. Mean total duration of illness was 9 months and almost half of the subjects were smokers. All the patients had HRCT pattern consistent with diagnosis of IPF and none of them had any co-morbid illnesses in the form of emphysema, Tuberculosis, malignancies, cardiac, renal, hepatic disorders and were negative for relevant collagen profile.

Details of physiological parameters are depicted in Table 2 which includes $6 \mathrm{MWD}$, Desaturation during $6 \mathrm{MWT}, \mathrm{FVC}, \mathrm{DL}_{\mathrm{CO}}$ and grading of dyspnea at the time of presentation and at the end of 6 months of standard regimen. All the patients except one (case no 15) had mild to moderately advanced disease in terms of FVC and were thus eligible candidates for pirfenidone therapy. 6 patients had an increase in 6MWD (ranging from 12 to 100 meters). 8 patients recorded fall in $6 \mathrm{MWD}$ (ranging from 8 to 52 meters). Irrespective of these figures, none of our patients recorded an improved $\mathrm{FVC}, \mathrm{DL}_{\mathrm{CO}}$ or grading of dyspnea at the end of 6 months. Except one patient (case no 12), all others also recorded an increase in desaturation during 6MWT at the end of 6 months (Table3 and Figure 1).

Table 1. Demographic profile of patients with IPF

\begin{tabular}{|c|c|}
\hline Total number of patients & 17 \\
\hline Patients completed study & 14 \\
\hline Mortality & 03 \\
\hline Male(12):Female(5) & $2.4: 1$ \\
\hline Mean age & 63 years \\
\hline Mean total duration of illness & 9 months \\
\hline Smoker & $9 / 17(53 \%)$ \\
\hline Co morbidities & None \\
\hline Collagen profile & All negative \\
\hline
\end{tabular}

Importantly Table 4 and Figure 2 shows that 13 out of 14 patients had grade II dyspnea at the time of initial presentation which progressed to grade III or IV at the end of 6 months.

Of note, an observation of immense importance is that all the three patients who died had $10 \%$ or more fall in saturation during $6 \mathrm{MWT}$ at the time of initial presentation. These patients also had the lowest FVC and lowest $6 \mathrm{MWD}$. Mean $\mathrm{DL}_{\mathrm{CO}}$ of these patients was also the lowest i.e. $30.75 \%$. Although very early to comment on outcome and not an intend of this study, at the end of 6 months, we observed that 7 patients (41\%) were stable, another 7 patients (41\%) progressively deteriorated and 3 patients (18\%) died.

\section{Discussion}

It is important to learn from various studies that long term survival was not linked to the underlying histological pattern of the disease (i.e. NSIP or UIP) but was solely dependent on the changes of lung physiology [8,9]. The ATS guidelines 2013[5] also suggests that in subjects with ILD, a reduction in $\mathrm{FVC}$ of at least $10 \%$ or $\mathrm{DL}_{\mathrm{CO}}$ of at least $15 \%$ over a period of 6-12 months is associated with reduced survival. Therefore, it is important to realize that a 6 monthly follow up of these patients with complete pulmonary function testing is of paramount importance. While plenty of literature is available on ILD and its corelation to FVC and $\mathrm{DL}_{\mathrm{CO}}$ [10-15], 6MWT has not much been studied with special reference to oxygen saturation and desaturation during the test. The CAPACITY and ASCEND Trials assessed the efficacy of pirfenidone in varying doses and compared with placebo. These studies revealed a reduced rate of decline in lung function with use of pirfenidone which was also associated with reduction in risk of death and disease progression [16,17]. A reduced decline in 6MWD was also noted. Similar results were also reported by Jeffery J Swigris et al. [18] and Essam et al. [19].

Our findings are similar to previous studies, where we found that overall mean distance walked by the patients during 6MWT did not change significantly (5.41 meters; $p$ value 0.967 ). However, in our study all three patients who died had a 6MWD of 126 meters or less suggesting that baseline low 6MWD might also have prognostic implications.

In contrast, a study by Roland $\mathrm{M}$ du Bois et al. [20] have clearly demonstrated that a decline of greater than 50 meters (6MWD) over 24 weeks had a fourfold increase in the risk of death at 1 year, suggesting that $6 \mathrm{MWT}$ is an important measure of prognosis and might be used as a key physiological outcome parameter. According to them, 6MWT might provide important incremental prognostic information, in addition to change in FVC which is considered a strong independent predictor of mortality in patients with IPF.

Therefore, from varying opinion in different studies, it can be concluded that $6 \mathrm{MWD}$ cannot be a sole parameter to explain prognosis of patients with UIP pattern specially when they are on pirfenidone therapy.

In our opinion, recording of $\mathrm{O}_{2}$ saturation at the beginning and degree of desaturation during 6MWT in follow up should be the other vital parameters to be monitored apart from $6 \mathrm{MWD}$. We observed that the mean desaturation after $6 \mathrm{MWT}$ at time of presentation was $6.35 \%$ and it increased to $9.64 \%$ at the end of 6 months. The difference between the mean was $4.64 \%$ and $p$ value was 0.001 which was statistically significant. Similar results were reported by Caminati et al. [21] in their patients of UIP which were followed for 12 months. They concluded that fall of saturation is the best predictor of prognosis. Liela et al. [22] also reported that $\mathrm{O}_{2}$ desaturation during 6MWT may be another 
Table 2. 6MWT and Physiologic profile of patients studied

\begin{tabular}{|c|c|c|c|c|c|c|c|c|c|c|}
\hline \multirow[t]{2}{*}{ S. No. } & \multicolumn{2}{|c|}{ Distance covered } & \multicolumn{2}{|c|}{ Desaturation (\%) } & \multicolumn{2}{|c|}{ FVC (\%) } & \multicolumn{2}{|c|}{ DLco (\%) } & \multicolumn{2}{|c|}{ Grading of dyspnea } \\
\hline & 0months & 6months & 0months & 6 months & 0months & 6months & 0months & 6months & 0 months & 6months \\
\hline 1 & 126 & 142 & 8 & 12 & 64 & 60 & 37 & 31 & 3 & 3 \\
\hline 2 & 147 & 110 & 8 & 12 & 72 & 67 & 42 & 30 & 2 & 4 \\
\hline 3 & 136 & 152 & 2 & 2 & 93 & 90 & 60 & 44 & 2 & 3 \\
\hline 4 & 105 & - & 10 & - & 42 & - & 35 & - & 3 & - \\
\hline 5 & 147 & 112 & 4 & 12 & 71 & 50 & 42 & 30 & 2 & 4 \\
\hline 6 & 147 & 132 & 5 & 10 & 97 & 84 & 52 & 41 & 2 & 4 \\
\hline 7 & 146 & 126 & 0 & 7 & 69 & 54 & 50 & 38 & 2 & 2 \\
\hline 8 & 122 & 147 & 7 & 20 & 60 & 48 & 35 & 16 & 2 & 4 \\
\hline 9 & 210 & 158 & 5 & 12 & 57 & 48 & 38 & 25 & 2 & 3 \\
\hline 10 & 126 & - & 13 & - & 53 & - & 31 & - & 3 & - \\
\hline 11 & 148 & 172 & 4 & 8 & 60 & 52 & 58 & 45 & 2 & 3 \\
\hline 12 & 170 & 270 & 3 & 0 & 91 & 83 & 37 & 30 & 2 & 3 \\
\hline 13 & 160 & 152 & 5 & 10 & 80 & 74 & 48 & 40 & 2 & 3 \\
\hline 14 & 168 & 160 & 7 & 10 & 93 & 88 & 33 & 23 & 2 & 3 \\
\hline 15 & 86 & - & 15 & - & 38 & - & 25 & - & 2 & - \\
\hline 16 & 158 & 134 & 9 & 12 & 59 & 53 & 46 & 28 & 2 & 3 \\
\hline 17 & 146 & 158 & 3 & 5 & 66 & 65 & 53 & 53 & 2 & 3 \\
\hline
\end{tabular}

Table 3. Mean, Percentage of difference and $\mathrm{p}$ value of various functional parameters at 0 and 6 months. Mean DLco in patients who died was $30.75 \%$

Mean desaturation in patients who died was $12.66 \%$

\begin{tabular}{|c|c|c|c|c|}
\hline \multirow{2}{*}{} & \multicolumn{2}{|c|}{ Mean \pm SD } & Mean Difference & P value \\
\cline { 2 - 5 } & 0 months & 6 months & 11.214 & $<0.001$ \\
\hline DLco & $42.47 \pm 9.900$ & $33.86 \pm 10.007$ & 8.286 & $<0.001$ \\
\hline FVC & $68.53 \pm 17.565$ & $65.43 \pm 15.649$ & -4.429 & 0.001 \\
\hline Desaturation & $6.35 \pm 3.904$ & $9.43 \pm 4.941$ & 0.429 & 0.967 \\
\hline Distance & $144.00 \pm 27.604$ & $151.79 \pm 38.579$ & & \\
\hline
\end{tabular}

Table 4. NYHA grading of study population

\begin{tabular}{|c|c|c|c|}
\hline NYHA Grading & 0 months & 6 months & P value \\
\hline 1 & - & - & \multirow{2}{*}{0.001} \\
\hline 2 & $13(45 \%)$ & $1(3 \%)$ & \\
\hline 3 & $1(3 \%)$ & $9(31 \%)$ & \\
\hline 4 & - & $4(14 \%)$ & \\
\hline
\end{tabular}

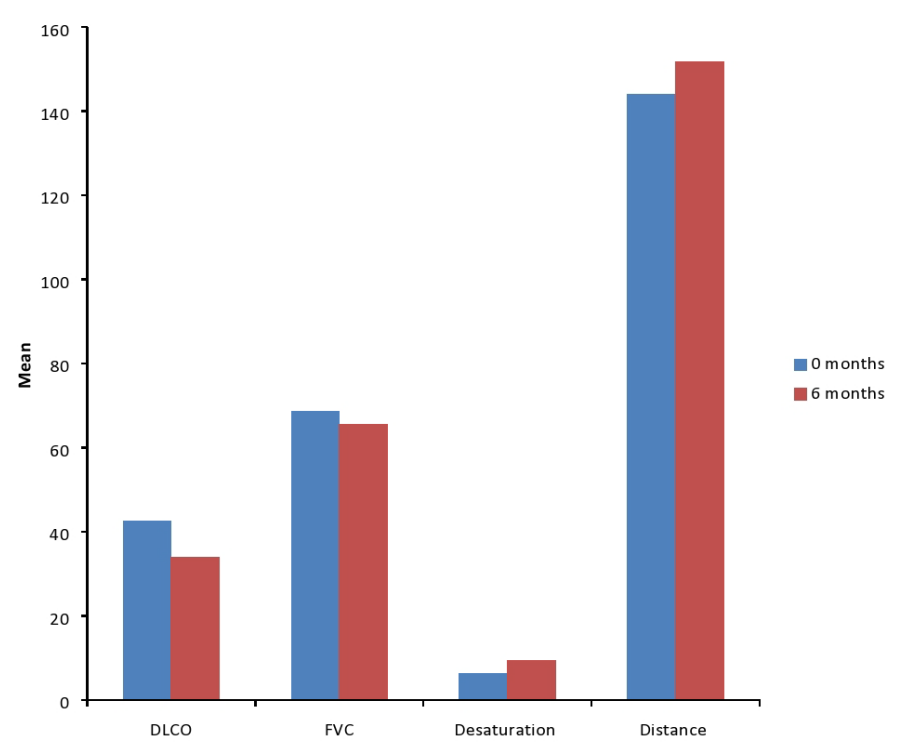

Figure 1. Graphical Observation of all the parameters studied at 0 month and end of $6^{\text {th }}$ month significant prognostic marker in patients with IPF. According to them, patients with oxygen saturation lower than $88 \%$ during the test have a higher mortality rate, a fourfold increase of mortality is probable in these patients during 3 years follow up. Lance et al. [11] demonstrated a strong correlation of measures of fall in saturation on 6MWT and survival, independent of the format used to define desaturation. Patients who desaturate had a higher mortality than patient who did not desaturate.

Antonella Caminati [23] stated that desaturation during 6MWT was not predictive of mortality in multivariate analysis. However, they observed that the only physiologic parameter that was predictive of survival over time was basal oxygen saturation suggesting that stability of PFTs might not reflect period of disease quiescence. One cannot deny that all 3 patients who died during follow up in our study had a significant fall in oxygen saturation during baseline 6MWT (>10\%).

This statistically significant increase in value of desaturation during 6MWT closely followed the statistically significant decline in FVC, DLco and grading of dyspnea in our patients (Table 3).

NYHA grading of dyspnea may be another important clinical parameter to be observed in these patients. Our observation with NYHA grading reconfirm that $6 \mathrm{MWD}$ did not have any impact on subject's perception of breathlessness. In most of our patients (12 out of 14), we observed a down grading (deterioration) of breathlessness scale despite the fact that the mean distance covered did not change significantly (Figure 2). We observed a statistically significant progression of dyspnea scale in our patients ( $\mathrm{p}$ value 0.001 ). To the best of our knowledge, there are no similar observations in the literature till now with new NYHA classification of dyspnea.

The fall in FVC, increased desaturation, reduction in diffusion capacity are ultimately reflected clinically by downgrading of NYHA dyspnea scale. Why these parameters did not correlate with 6MWD is not clear. A possible explanation given by Essam H Alhamad [19] sounds scientifically relevant. He hypothesised that skeletal muscle weakness (i.e acquired myopathy), which is frequently observed in IPF patients due to hypoxia, stress, malnutrition, corticosteroid therapy and other factors, is associated with increased activity of transforming 
NYHA

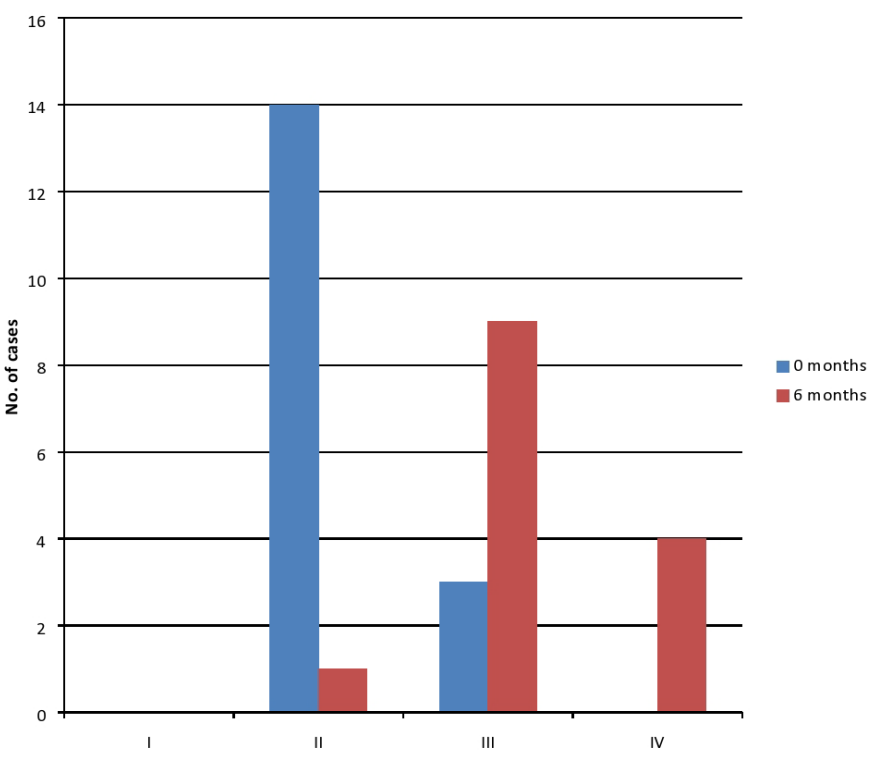

Figure 2. Graph showing pattern of NYHA grading at 0 and 6 months

growth factor beta (TGF- $\beta$ ). Because the anti-fibrotic properties of pirfenidone are mediated by the inhibition of TGF- $\beta$ expression, as observed in many models of fibrosis, including in the lung, heart, liver and kidney, such actions may also occur in skeletal muscles, resulting in improved functional capacity and exercise tolerance in patients with IPF. However, this hypothesis is only speculative, and future studies are needed to address the precise role of pirfenidone on TGF- $\beta$ signaling in skeletal muscles and possible subsequent effect on 6MWD.

The main constraint in our study was smaller sample size of population studied but we could not resist ourselves to share our observations which posed very indispensable to us. The study is still ongoing and we will like to update it in future with a bigger sample size and more promising and well-founded results.

\section{Conclusion}

Our observations and correlating them with previous studies, we are of the opinion that-

I. NYHA dyspnea grading must be a part of clinical examination in patients of IPF. It closely correlates with other pulmonary function parameters.

II. 6MWT is still a very helpful, simple, noninvasive test to evaluate exercise capacity of patients with IPF. However, $6 \mathrm{MWD}$ cannot be solely relied upon as a prognostic factor but measurement of baseline oxygen saturation and desaturation during 6MWT and follow up studies must be incorporated in evaluation of these patients.

III. Facilities of spirometry and diffusion capacity may not be universally available, in that case NYHA grading and 6MWT must suffice to prognosticate the patient.

\section{References}

1. Solway S, Brooks D, Lacasse Y, Thomas S (2001) A qualitative systematic overview of the measurement properties of functional walk tests used in the cardiorespiratory domain. Chest 119: 256-270. [Crossref]

2. Guyatt GH, Townsend M, Keller J, Singer J, Nogradi S (1991) Measuring functional status in chronic lung disease: Conclusions from a random control trial. Respir Med 85(suppl B): 17-21. [Crossref]
3. Niedermann MS, Clemente PH, Fein AM, Feinsilver SH, Robinson DA, et al. (1991) Benefits of a multidisciplinary pulmonary rehabilitation programme: Improvement are independent of lung function. Chest $99: 798-804$. [Crossref]

4. Noseda A, Carpianx J, Prigogine T, Schmerber J (1989) Lung function, maximum and submaximum exercise testing in COPD patients: reproducibility over a long interval. Lung 167:247-257. [Crossref]

5. Travis WD, Costabel U, Hansell DM, King TE Jr, Lynch DA, et al. (2013) An official American Thoracic Society/European Respiratory Society statement: Update of the international multidisciplinary classification of the idiopathic interstitial pneumonias. Am RespCrit Care Med 188: 733-748. [Crossref]

6. ATS Committee on Proficiency Standards for Clinical Pulmonary Function Laboratories (2002) ATS statement: guidelines for the six-minute walk test. Am J RespirCrit Care Med 166: 111-117. [Crossref]

7. The criteria committee of New York Heart Association (1994) Nomenclature and Criteria for Diagnosis of Diseases of the Heart and Great vessels. 9th ed. Boston, Mass: Little, Brown \& Co $p p: 253-256$.

8. Jegal Y, Kim DS, Shim TS, Lim CM, Do Lee S, et al. (2005) Physiology is a stronger predictor of survival than pathology in fibrotic interstitial pneumonia. Am J RespirCrit Care Med 171: 639-644. [Crossref]

9. Latsi PI., du Bois RM, Nicholson AG (2003) Fibrotic idiopathic interstitial pneumonia: the prognostic value of longitudinal functional trends. Am J RespirCrit Care Med168: 531-537. [Crossref]

10. O’Donnell D (1998) Physiology of interstitial lung disease. In: Schwarz M, King T $\mathrm{Jr}$ (editors). Interstitial lung disease. Hamilton, ON, Canada: Marcel Dekkerpp:51-70.

11. Lama VN, Martinez FJ (2004) Resting and exercise physiology in interstitial lung diseases. Clin Chest Med 25: 435-453, v. [Crossref]

12. Jezek V, Fucik J, Michaljanic A, Jeskova L (1980) The prognostic significance of functional tests in cryptogenic fibrosingalveolitis. Bull EurPhysiopatholRespir16: 711720. [Crossref]

13. Raghu G, Johnson W, Lockhart D, Mageto Y (1999) Treatment of idiopathic pulmonary fibrosis with a new antifibrotic agent, pirfendone: results of a prospective, open-label phase-2 study. Am J RespirCrit Care Med159: 1061-1069. [Crossref]

14. Novelli F, Tavanti L, Cini S, Aquilini F, Melosini L, et al. (2014) Determinants of the prognosis of idiopathic pulmonary fibrosis. Eur Rev Med PharmacolSci 18: 880-886. [Crossref]

15. Hanson D, Winterbauer R, Kirtland S, Wu R (1995) Changes in pulmonary function test results after 1 year of therapy as predictors of survival in patients with idiopathic pulmonary fibrosis. Chest 108: 305-310. [Crossref]

16. Noble PW,Albera C, Bradeford WZ, Costable U, Glassberg MK, et al. (2011) Pirfenidone in Patients with Idiopathic Pulmonary Fibrosis(CAPACITY): Two randomised trials. Lancet377: 1760-1769. [Crossref]

17. King TE Jr, Bradford WZ, Castro-Bernardini S, Fagan EA, Glaspole I, et al. (2014) A phase 3 trial of Pirfenidone in patients with Idiopathic Pulmonary Fibrosis. $N$ Engl $J$ Med370: 2083-2092. [Crossref]

18. Swigris JJ, Brown KK (2006) Idiopathic pulmonary fibrosis: a decade of progress. $J$ Bras Pneumol32: 249-260. [Crossref]

19. Alhamad EH (2015) Pirfenidone treatment in idiopathic pulmonary fibrosis: A Saudi experience. Ann Thorac Med 10: 38-43. [Crossref]

20. du Bois RM, Derek W, Carlo A, Williamson ZB, Ulrich C, et al. (2011) Six Minute walk test in Idiopathic Pulmonary Fibrosis.Am J Resp and Crit Care Med183: 12311237. [Crossref]

21. Caminati A, Bianchi A, Cassandro R, Mirenda MR, Harari S (2009) Walking distance on 6-MWT is a prognostic factor in idiopathic pulmonary fibrosis. Respir Med 103 : 117-123. [Crossref]

22. Leila G, Zahra DS, Farrneh V, Mohamad K, Zahra JA (2015) Six Minute Walk Test for Evaluation of Pulmonary diseases. J Cardio Thoracic Med3: 284-287.

23. Caminati A, Bianchi A, Cassandro R, Mirenda MR, Harari S (2009) Walking distance on 6-MWT is a prognostic factor in idiopathic pulmonary fibrosis. Respir Med 103 117-123. [Crossref]

Copyright: (C2017 Gupta N. This is an open-access article distributed under the terms of the Creative Commons Attribution License, which permits unrestricted use, distribution, and reproduction in any medium, provided the original author and source are credited. 